\title{
An Investigation into the Exploratory Use of Additive Manufacturing in Weir Design and Open Channel Flow
}

\author{
Robert BENHAM ${ }^{1}$ and Fayyaz REHMAN \\ Faculty of Creative Industries, Architecture and Engineering, Southampton Solent \\ University, Southampton, Hampshire, SO14 OYN, UK
}

\begin{abstract}
Additive Manufacturing (AM) offers a range of possibilities in fluid flow research. An existing $2.5 \mathrm{~m}$ open channel fluid flow experiment contains a set of standard weirs which are limited in design. This research will compare experimental AM weirs (e.g. labyrinth, piano, catenary), that would not be possible on some laser-cut polymer or machined aluminium weirs. Due to the bespoke complex nature of weirs' design other manufacturing methods would be too expensive and impossible to use. AM technology allows a cost-effective solution for progressive design modifications to be implemented throughout investigations. This paper will highlight comparisons made between a range of AM produced weirs in terms of flow rate, fluid velocity profile, water level height and discharge coefficient. Computation fluid dynamic modelling (CFD) will also be used to verify, analyse, and compare results. Based on the experimental results and verification, the paper will also discuss the suitability of application of AM techniques in fluid flow analysis.
\end{abstract}

Keywords: Experimental methods, additive manufacturing, photopolymer resin.

\section{Introduction}

Weir improvements have historically required a substantial amount of empirical testing. In addition, computational fluid dynamic (CFD) modelling can be used to simulate the flow and predict parameters that cannot be estimated accurately from the experiment. Conventional testing for open channels utilised sheet polymers which are cut either manually or by laser cutting. Additive manufacturing (AM) printed parts offer several distinct advantages over conventionally cut components, in terms of production time, cost, and the possibilities of achieving more complex profiles. The existing experiment used at the University for undergraduate students studying Mechanical Engineering uses a $2.5 \mathrm{~m}$ long flow channel, a hydraulic tank and a selection of standard machined weirs and other attachments. The flow channel can be used to test more complex geometry parts. Multiple weirs can be created with differing geometries at a low cost and fabricated in hours. During the experiment students record a range of volumetric flow rates and determine key fluid parameters.

\footnotetext{
${ }^{1}$ Corresponding Author. robert.benham@solent.ac.uk
} 


\section{Methodology}

New weirs are made from tough additive manufacturing polymer, like ABS, a translucent plastic type commercially available photopolymer resin using a Stereolithography (SLA) machine. Due to the limited time and preliminary testing, the number of weirs was restricted to four models that could be compared to the supplied machined sharp-crested weir. The weirs selected for this study include two with a catenary crest (one empty and one ramped), a labyrinth weir and a piano key type weir, which are shown in Figure 1. To improve the flow, the flow rate and reduce the difference in upstream head the length of the crest can be increased.

Two flow rates were used: maximum flow (to mimic flood conditions) and minimum flow (ensuring full coverage of the weir wall). The datum for taking height measurements was the top of the flow channel and depth to the water level was measured by a depth gauge. Points were measured at $200 \mathrm{~mm}$ intervals close to the weir upstream and $600 \mathrm{~mm}$ from the weir downstream. A pitot tube connected to a data logger was used to measure the velocity and a representative value was recorded at each of the measuring points using the statistics tool over 20 -second time periods.

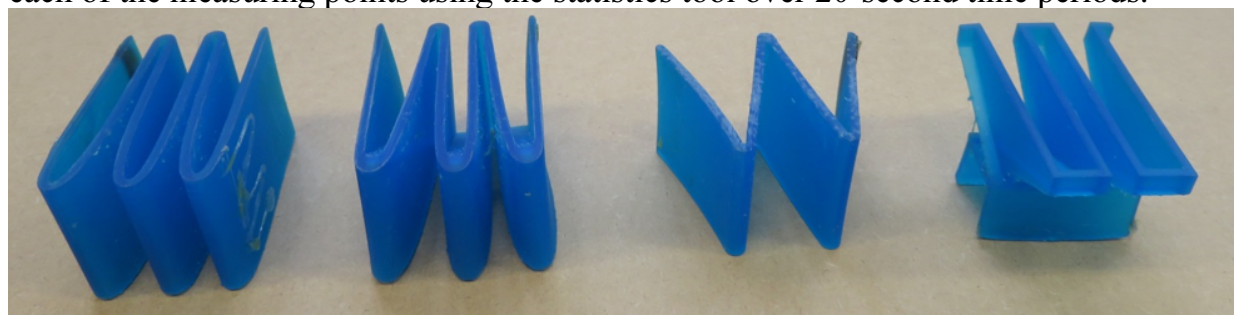

Figure 1. AM weirs (from left to right: empty catenary, ramped catenary, labyrinth, piano key).

Each weir had a flat crest; further research will look at variations in crest profile. The flow channel was run empty and recorded when set at $0.5^{\circ}$ at full flow. A number of parameters were calculated, such as volumetric flow rate, average velocity, the Reynolds number, Froude number. The weirs were then tested at maximum flow rate and then a reduced flow rate so that the range in upstream height could be calculated and the weirs could be compared under different flow conditions. Computational Fluid Dynamic (CFD) modelling based on flow simulation was carried out using the actual flow rates, so that the variations in velocity profile could be seen and regions of interest established.

A spreadsheet was used to compute the flow rates, Froude and Reynolds numbers as well as carry out comparisons to the supplied (machined) sharp-crested weir. The main comparison factors used are: the average height of the water marks upstream; the differences in water heights between the maximum and minimum flow rates; the discharge enhancement ratio, referred to here as the " $r$ " value (see equation 1 ); the mass-flow rate ratio of discharge between the upstream and downstream and the mass flow rate downstream. CFD modelling was used to compare the calculated the parameters and in addition observe the velocity profiles at regions in the channel such as the weir approach, the crest, nappe, and other sections of the channel far away from the weir. 


\section{Analysis of Results}

One factor that limits the testing the relatively narrow width of the channel $(53 \mathrm{~mm})$. A lot of research employs wider channels, although the results overall show that the AM weirs had good consistency for the upstream water height a maximum flow. One problem with geometry of the weirs coupled with the parameters was to try and determine the coefficient of discharge accurately. As mentioned by several authors, notably Kumar et al (2020) the determination of these parameters is complicated often requires different modelling approaches. Unlike the sharp-crested weir that has a relatively straight forward formula to calculate the coefficient of discharge, $C_{d}$, the other weirs could be compared instead by the discharge enhancement ratio:

$$
r=\frac{Q_{A M \text { weir }}}{Q_{\text {Sharp-crested weir }}}=\frac{C_{d} L_{\text {effective }} \sqrt{2 g} H^{\frac{3}{2}}}{C_{d} W \sqrt{2 g} H^{\frac{3}{2}}}=\frac{L_{\text {effective }}}{W}
$$

Initial testing of the weirs showed very clearly that variation in upstream height between the maximum and minimum flow rates was much lower than the sharp-crested weir. Another aspect that can be easily compared is the gradient of the water line over the side length of the weir (figures 2 and 3), and the difference in forms of nappes at the flow rates. The labyrinth weir did not compare well in on most of the comparison criteria for both flow rates, however the major limitation of only having two cycles meant that weir characteristics are unlikely to be similar for much wider channels. Labyrinth weirs are attracted a lot of interest due to their relatively long existence and widespread use; studies by Crookston, Paxson and Campbell (2013) do compare several geometrical factors and include a 2-cycle labyrinth weir to others. A logical next step in this research would be compare different crest profiles (e.g., sharp, flat, quarter-round, half-round, ogee). The catenary shape derived from the hyperbolic cosine function $(\cosh x)$ was chosen by authors due to the relatively long crest that can be achieved in a relatively narrow channel.

One issue that could be improved in terms of design for testing AM parts is reducing the side wall and under some weirs, this was most noticeable with the piano key weir. The most effective weir preventing this was the ramped catenary weir, due to the increased rigidity of the model.

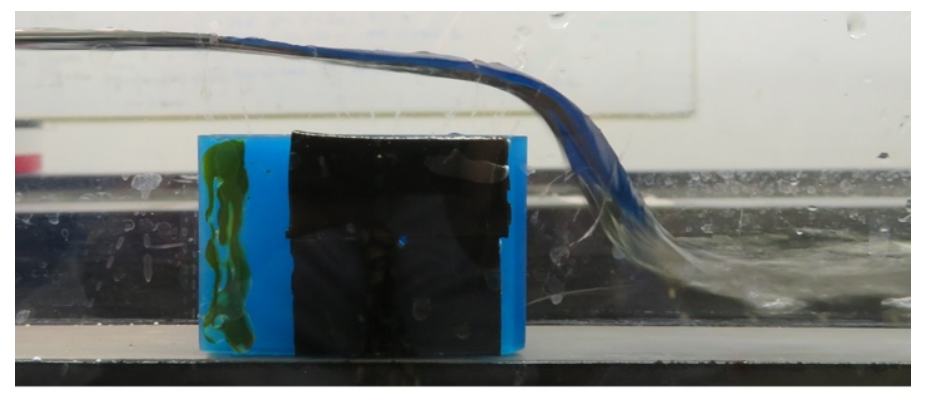

Figure 2. Side-on view of the empty catenary weir at full flow rate. 


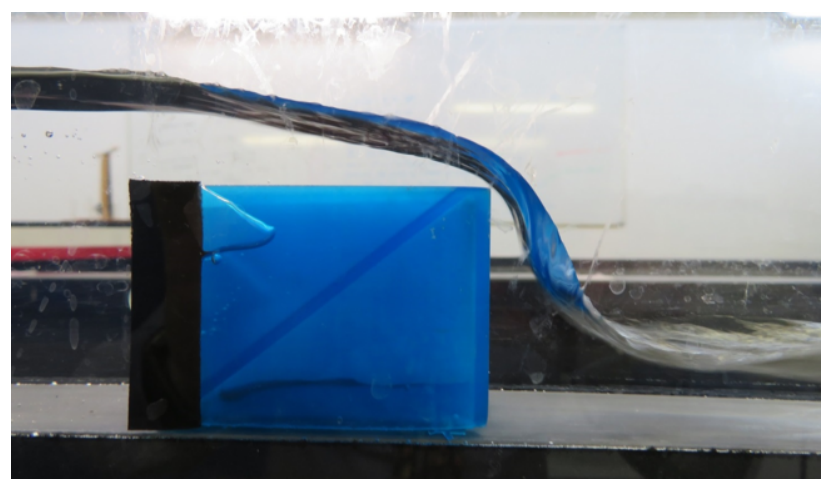

Figure 3. Side-on view of the ramped catenary weir at full flow rate.

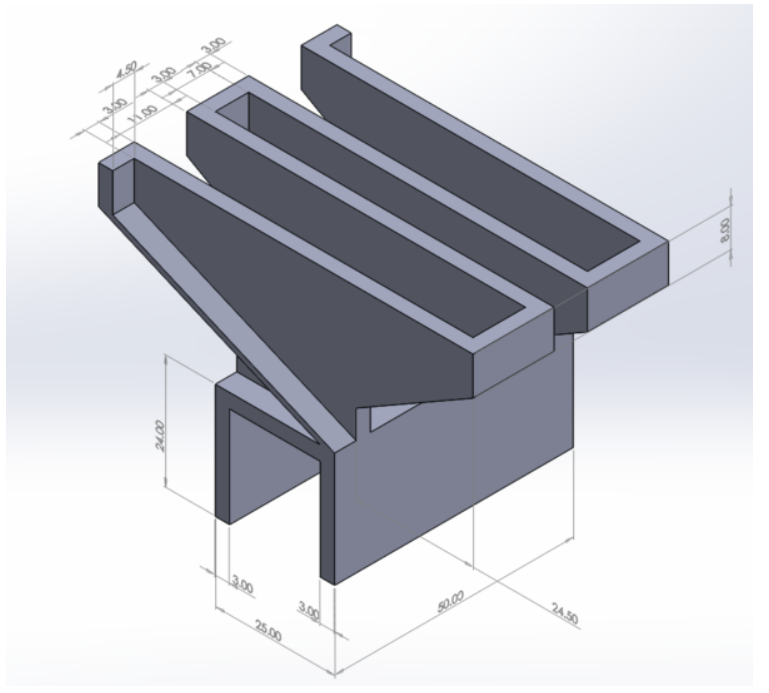

Figure 4. The piano key weir design for the flow channel.

The piano key weir (figure 4) design was based on similar design ratio parameters from other research (Erpicum et al), and at high flow rates scored well. The most consistent in terms of ranking over all comparators is the ramped catenary, in third place at maximum flow but second at lowest flow. The error of the pitot tube was evaluated in the empty channel, where velocity was calculated from the volumetric flow rate. It was found to be $96 \%$ accurate, from the readings taken.

\subsection{Flow simulation-based results evaluation}

Finite Element Analysis based CFD flow simulations were done to evaluate and compare the results obtained from experimental data for all four different types of weirs. Flow simulations accurately predicted the pattern of fluid flow as well as well as calculation of average velocities. The fluid flow pattern observed through CFD analysis 
of both empty (Figure 5) and ramped (Figure 6) catenary weirs is almost like experimental flow pattern.

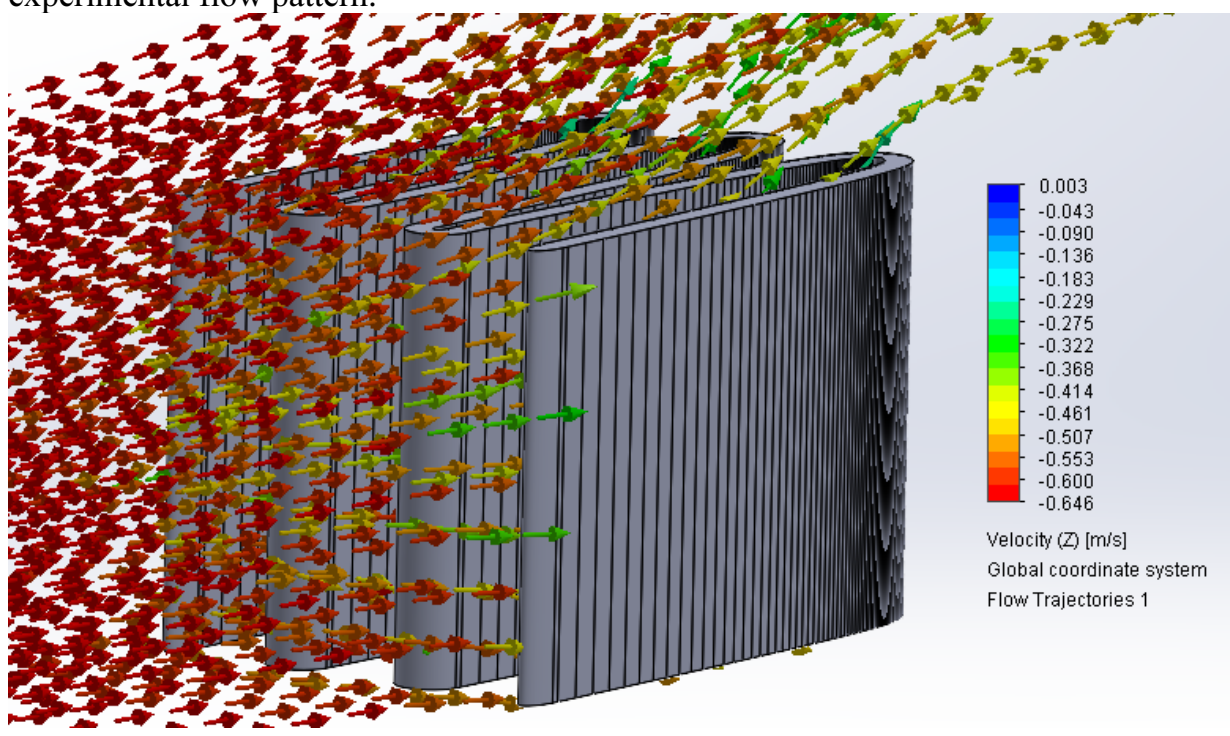

Figure 5. Flow simulation of empty catenary weir.

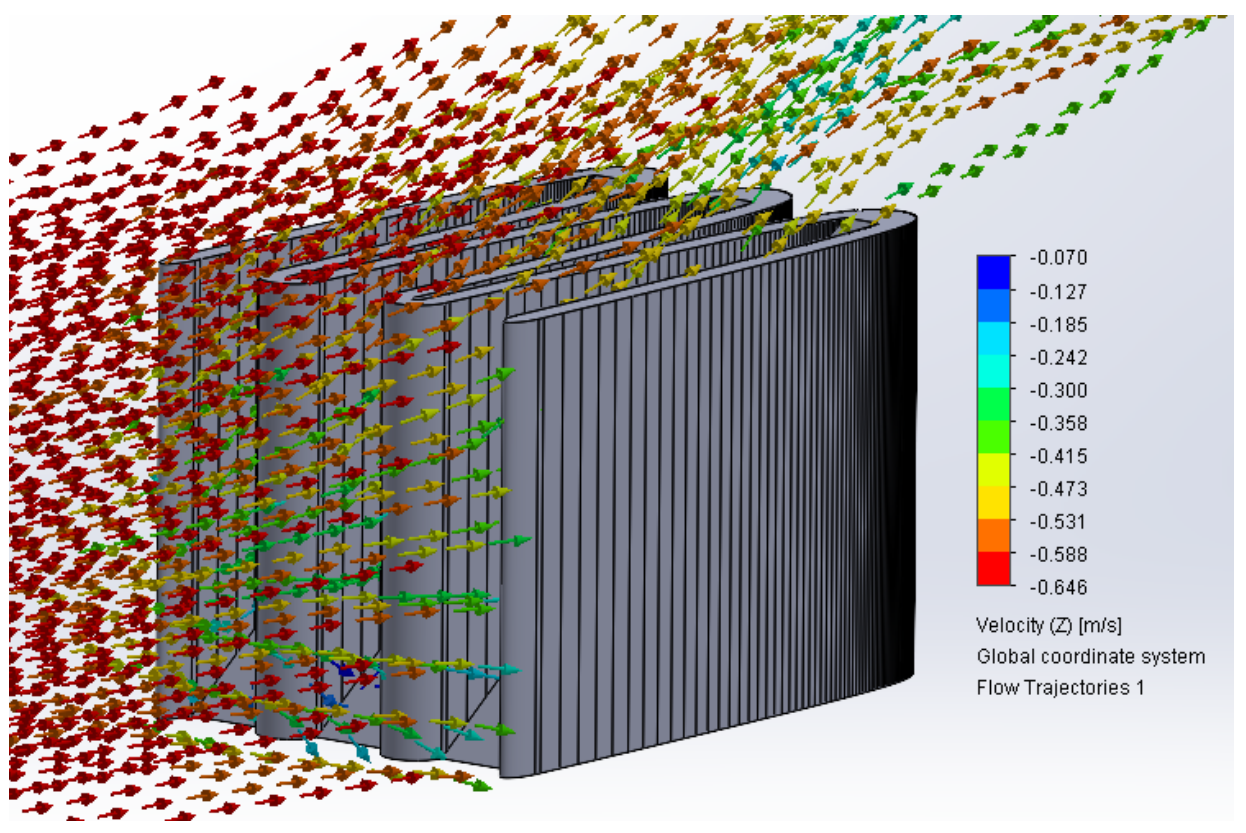

Figure 6. Flow simulation of ramped catenary weir

Simulation results also verified and compared the average velocities obtained from experimental data for both empty and ramped catenary weirs shown in Figure 7. 
Change in shape from empty to ramped shaped weir negligible reduction in average velocities.

\begin{tabular}{|c|c|c|}
\hline Type of Catenary Weir & $\begin{array}{c}\text { Experimental Average } \\
\text { Velocity (m/sec) }\end{array}$ & $\begin{array}{c}\text { CFD Average Velocity } \\
\text { (m/sec) }\end{array}$ \\
\hline Empty & 0.650 & 0.628 \\
\hline Ramped & 0.650 & 0.627 \\
\hline
\end{tabular}

Figure 7. Comparison of Experimental/Simulation Data for average velocities in both catenary weirs.

\section{Conclusions}

The research shows that effective weir designs can be implemented and compared using suitable AM technologies. The tough resin performs well although the flexibility in some designs requires further structural rigidity around the base to prevent the deformation of the shape when held in the channel. The catenary weirs show promising results however the regions of interference need to be studied further, and extra features could be added to try and reduce the detrimental factors on performance. For example, nappe breakers have been experimented with in several studies, Bilan et al (2018), and AM can allow for more complex geometries to be investigated. Further investigation is required prior to this to compare different forms of crest, as the flat crest is not optimal. In conjunction with this, coefficients of discharge need to be mathematically determined and compared to the measured flow rates over the weir. The volumetric flow rates can then be compared against the changes in height upstream to give a better comparison. The relatively narrow channel does limit the amount of work that can be carried out; however, the results show that even with flat crested weirs the variation in weir design can be effectively compared by using AM technology, which can give a good advantage over other construction methods of model weirs for open channel flow studies. The weirs created for this study will be used alongside the supplied weirs for student laboratory work in the future and will enhance the laboratory testing furthermore.

\section{References}

[1] Kumar, M., Sihag, P., Tiwari, N. K., \& Ranjan, S. (2020). Experimental study and modelling discharge coefficient of trapezoidal and rectangular piano key weirs. Applied Water Science, 10(1), 1-9.

[2] Crookston, B., Paxson, G., \& Campbell, D. (2013). Effective spillways: Harmonizing labyrinth weir hydraulic efficiency and project requirements. Labyrinth and Piano Key Weirs II, 283.

[3] Erpicum et al, Labyrinth and Piano Key Weirs. (2011). United Kingdom: Taylor \& Francis.

[4] Bilhan, O., Emiroglu, M. E., Miller, C. J., \& Ulas, M. (2018). The evaluation of the effect of nappe breakers on the discharge capacity of trapezoidal labyrinth weirs by ELM and SVR approaches. Flow Measurement and Instrumentation, 64, 71-82. 